\title{
The Tribological and Sealing Properties of PFA Composites
}

\author{
Ye Sujuan $\mathbb{D}^{1}{ }^{1}$ Yu Quan, ${ }^{2,3}$ Li Kunquan, ${ }^{2}$ Tan Feng, ${ }^{1}$ Fan Qing, ${ }^{1}$ Tan Guibin, ${ }^{1}$ \\ and Huang Xing ${ }^{1}$ \\ ${ }^{1}$ Guangzhou Mechanical Engineering Research Institute Co., Ltd., Guangzhou, Guangdong 510700, China \\ ${ }^{2}$ Kingfa Sci. \& Tech. Co., Ltd., Guangzhou, Guangdong 510670, China \\ ${ }^{3}$ College of Materials Science and Engineering, South China University of Technology, Guangzhou, Guangdong 510640, China
}

Correspondence should be addressed to Ye Sujuan; yesujuan100@163.com

Received 21 November 2017; Accepted 26 April 2018; Published 16 May 2018

Academic Editor: De-Yi Wang

Copyright (c) 2018 Ye Sujuan et al. This is an open access article distributed under the Creative Commons Attribution License, which permits unrestricted use, distribution, and reproduction in any medium, provided the original work is properly cited.

The polytetrafluoroethylene (PTFE) and perfluoroalkoxyperfluoropropyl vinyl ether (PFA) composites were prepared using carbon fiber (CF) and glass fiber (GF) as fillers. The effects of these fillers on the tribological and sealing properties were investigated. The tribological and sealing properties of the composites were evaluated by a wear tester and a seal test rig. Results showed that the incorporation of CF and GF both improved the wear resistance with composites, approaching wear rates as low as $10^{-6} \mathrm{~mm} / \mathrm{N} \cdot \mathrm{m}$ and lowered the seal's leakage of the PTFE/PFA composites. As described here, CF was more effective than GF in improving the wear rate. Notably, the wear rate of the PFA/CF composite is much less than that of the PTFE composites as the transfer film on the couple ring is much more unique, thick, smooth, and compact. The leakage of carbon fiber-filled PFA composite seal was lowered to $66 \%$ less than the traditional PTFE composite seals, which indicates better sealing properties.

\section{Introduction}

Seals are important elements in hydraulic devices, as some of the basic requirements are low friction and leakage near zero spanning the entire lifetime of the device. Low friction and leakage of seals are influenced by the seal's design, the counterparts' surface, lubrication conditions, and the tribological properties of the seal material. The properties of friction and wear of sealing materials are related to a seal's leakage and lifetime. The material of the seal itself will also influence the sealing characteristics. Other influences may include increased friction or energy expenditure and the possibility of leakage or failure, as well as the lifetime of the sealing system. There are several studies covering the topic of sealing technology, but only a few of them discuss the friction and wear properties of sealing materials.

PTFE is a well-recognized seal material due to its excellent properties, such as low coefficient of friction and good resistance to oils and oil additives. However, its unacceptably high wear rate has restricted its use in most applications. To solve high wear rate, various suitable fillers have been added to PTFE to improve the wear resistance. Most serve to moderately reduce wear (by one or two orders of magnitude) while increasing friction [1-4]. However, complex geometries of PTFE products must be machined after the part has been sintered, which adds cost to the manufacturing process. PFA is a melt-processable polytetrafluoroethylene with a small amount of perfluoropropyl vinyl ether (PPVE) added to allow its processing by injection molding. PFA exhibits nearly the same unique properties as PTFE since only a small amount of PPVE is added. PFA has a larger coefficient of friction than PTFE, but it is still lower than many bulk polymers and can be used for a wide range of operating temperatures $[5,6]$.

This work deals with the investigation and characterization of tribological properties of PTFE, PFA, and their composites. Special focus is given to the improvement of both friction and wear properties. Finally, the results of the tribological properties are compared with the seals' properties tested by a test rig. 


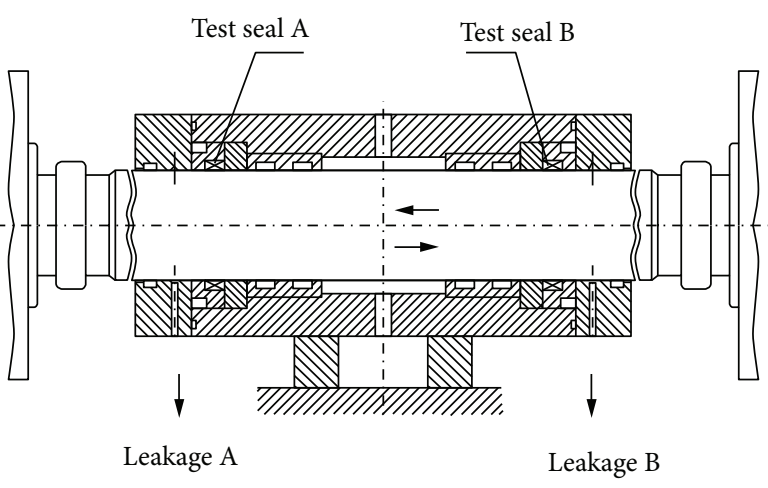

FIGURE 1: The sketch map of the reciprocating seal test.

\section{Experiments}

2.1. Materials. The PTFE (type: M18F) powder with a particle size of $25-40 \mu \mathrm{m}$ is supplied by Daikin Industries, Ltd. The PFA (340) is supplied by Chemours. Glass fiber is supplied by Nanjing Fiberglass R\&D Research Institute. Carbon fiber is supplied by Qingdao Yuan Hui Composite Material Co., Ltd.

2.2. Specimen Preparation. The carbon/glass fiber and PTFE were kept in an oven at $105^{\circ} \mathrm{C}$ for $3 \mathrm{~h}$ before the mixing process. The materials were then prepared and blended in a high-speed mixer. After mixing, the materials were preformed into a mold by means of compression at ambient temperature to yield a final compact form. The pressure used for the preform is $40 \mathrm{MPa}$. Next, the preform was removed from the mold and sintered. Composite samples were produced by free sintering in air at $380^{\circ} \mathrm{C}$ for 1 hour, using $40^{\circ} \mathrm{C} / \mathrm{h}$ heating and cooling rates until ambient temperature was reached. Finally, the samples were machined to produce samples for testing.

The carbon/glass fibers were kept in an oven at $105^{\circ} \mathrm{C}$ for $3 \mathrm{~h}$ prior to compounding. PFA pellets were used directly. Blends of PFA and glass fiber or carbon fiber were prepared using a twin screw mega compounder ZE 25 UTX UltraGlide with a typical screw design for dispersion of inorganics in fluorinated materials. PFA/GF or PFA/CF pellets were molded into samples by a single screw. $10 \mathrm{wt} . \%$ of GF or CF was filled into PFA and PTFE to form the composites.

2.3. Test Details. The tribological behaviors of composites when sliding the composites against a stainless steel ring were evaluated on an M-2000 (Zhangjiakou Xuan Hua Ke Hua Mechanical Machine Co., Ltd.) friction and a wear tester. The wear rate test at different temperatures was evaluated on CFT-I (Lanzhou Zhong Ke Kai Hua Keji Co., Ltd.) friction and a wear tester. The morphologies of the composite worn surfaces were examined with scanning electron microscope (SEM; JSM-6330F JEOL) and an optical microscope (Leica, M80, Germany). Samples were coated with a thin layer of gold to increase the electrical conductivity testing.

The test rig shown in Figure 1 was developed to determine the properties of the PTFE and PFA composite seals. The test rod is in the form of a step seal. A hard, chrome-plated, ground rod with a nominal diameter of



Figure 2: Coefficients of friction variation of the PTFE and PFA composites with respect to time.

$120 \mathrm{~mm}$ was used. The test procedure started with a velocity of $0.2 \mathrm{~m} / \mathrm{s}$ and a pressure of $31 \mathrm{MPa}$.

\section{Results and Discussion}

\subsection{Tribological Properties}

3.1.1. Coefficients of Friction. Figure 2 shows how the coefficients of friction of the PTFE and PFA composites vary with time. Results show that adding fillers to PTFE and PFA increased the coefficients of friction of the composites. The coefficients of friction of the PFA composites are somewhat higher than that of PTFE composites but more stable after the initial run-in period. After the initial run-in period, the coefficients of friction of the PFA composites are higher than those of the PTFE composites due to the significantly higher coefficient of friction of unfilled PFA compared to unfilled PTFE. Adding glass fibers to PTFE and PFA composites increased the coefficients of friction to a higher degree than adding carbon fiber. In comparison with PFA, PTFE and its composites are characterized by low coefficients of friction. The higher coefficients of friction of PFA and its composites may be attributed to a varyingly lower degree of crystallinity, which results in a tighter solid-state morphology and therefore enhanced adhesion to the counterpart.

3.1.2. Wear Resistance. The wear rates of the PTFE and PFA composites are presented in Figure 3. PFA shows an almost 30 times lower wear rate than PTFE, due to the presence of PPVE as a constituent unit in PTFE chains. The wear rate of PTFE is associated predominantly with the breaking of weak intermolecular bonds and with interplanar shear and slipping of crystalline aggregates formed in the band structure of materials. The presence of PPVE as the side group in PFA inhibits these processes, leading to noticeably improved wear resistance [7]. Results indicated that adding glass fiber or carbon fiber distinctly improved the wear resistance (almost 500 times lower wear rate) of both PTFE and PFA composites. The lower viscosity of PFA enables better dispersion results in the filler-matrix morphology which 


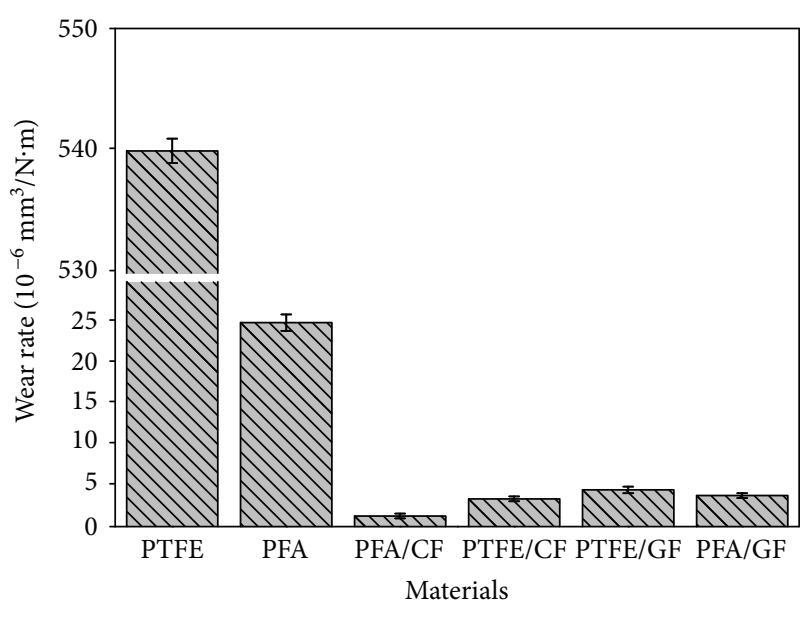

Figure 3: Wear rates of the PTFE and PFA composites.

reduces wear rates. One of the consistent findings in the determination of wear rate reduction is the formation of a better adhered, more continuous thin transfer film. The tribological properties of PTFE/PFA and their composites sliding against a metal under dry friction conditions are strongly influenced by their ability to form a transfer film on the counterpart surface. Once a transfer film is formed, subsequent interactions occur between the polymer and a layer of similar material, irrespective of the composition of the substrate [8-10].

Figure 4 shows the wear rates of the PTFE and PFA composites at different high temperature conditions. The results show that there is a correlation between wear rates and temperature, where both wear rates and temperature increase for the PTFE and PFA composites. As temperature increases, the PTFE and PFA composites become softer. This allows the coupling ring to plow the composites more easily, consequently increasing the wear rates. The wear rates of PFA composites are smaller than those of PTFE composites at any temperatures. Compared with PTFE, PFA has much better heat resistance and keeps better mechanical properties at high temperature, leading to better wear resistance. Lower wear rates signify that PFA composites can work better than PTFE composites at higher temperatures. The wear rates of PFA composites are lower than those of PTFE composites due to enhanced dispersion of CF or GF in PFA than in PTFE. Compared with PTFE, the presence of PPVE as the side group in PFA improved the PFA composites' interfacial strength and the overall dispersion of the fillers in the composites.

3.1.3. SEM of Worn Surfaces. Figure 5 shows the worn surface morphology of the PTFE and PFA composites. On the worn surface of pure PTFE, large-scale wear debris has collected and plow-like markings have formed. This contrasts the worn surfaces of the PTFE composites which are smoother than those of pure PTFE. In the case of the composites, there was small debris on the surface of PTFE and PFA composites. Compared to PTFE, the worn surface of PFA exhibits less wear debris and no indentations. Unfilled PFA delaminates,

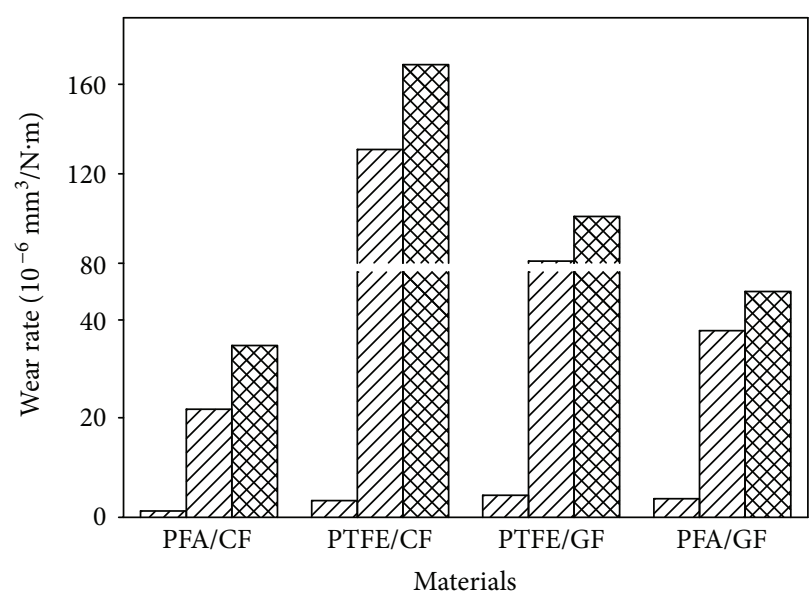

ZII $25^{\circ} \mathrm{C}$

ZII $100^{\circ} \mathrm{C}$

$150^{\circ} \mathrm{C}$

FIgUre 4: Wear rates of the PTFE and PFA composites at different temperatures.

generating large, plate-like debris, and forms a fragmented, poorly adhered transfer film on the counter surface. The wear debris particles of the composites are much smaller than those of pure PTFE and PFA. This is in agreement with Blanchet et al., who noted that wear debris was generated due to the disruption of nanometer-scale transfer films and thicker plates, lumps, and slabs of polymer formed on the counter face during the repetitive sliding action [11]. The reinforcing phases block the cracks that form in the unfilled regions, thus producing platelet debris approximately equal in size to the original compartment. Debris size reduction is therefore believed to be the wear reduction mechanism of the PTFE and PFA composites. The worn surfaces of PFA/ GF and PFA/CF composites are much smoother than those of PTFE composites.

3.1.4. Transfer Film of Counterparts. Figure 6 shows that sufficient transfer films between the PTFE/CF or PFA/CF composites and the couple surfaces that are formed from the operating conditions, while the pure PTFE and PFA lack transfer films. It can be inferred that PTFE/CF and PFA/CF composites can facilitate the formation of transfer films on the steel ring's surfaces and strengthen the bond between the transfer film and the counterpart surface, which results in much lower wear rate for the PTFE and PFA composites in comparison to pure PTFE or PFA. Transfer films are very important to wear resistance, especially for dry conditions. During sliding, when transfer films were formed, the tribological conditions were improved whereby composites would rub against themselves rather than the metal counterpart's surface. Results show that the transfer film of the PFA/CF composite is much more unique, thick, smooth, and compact when compared to that of the PTFE/CF composite. The wear rate of the PFA/CF composite will be much lower while this unique transform film is formed as the PFA/CF materials rub against themselves. The formation of a stable, 

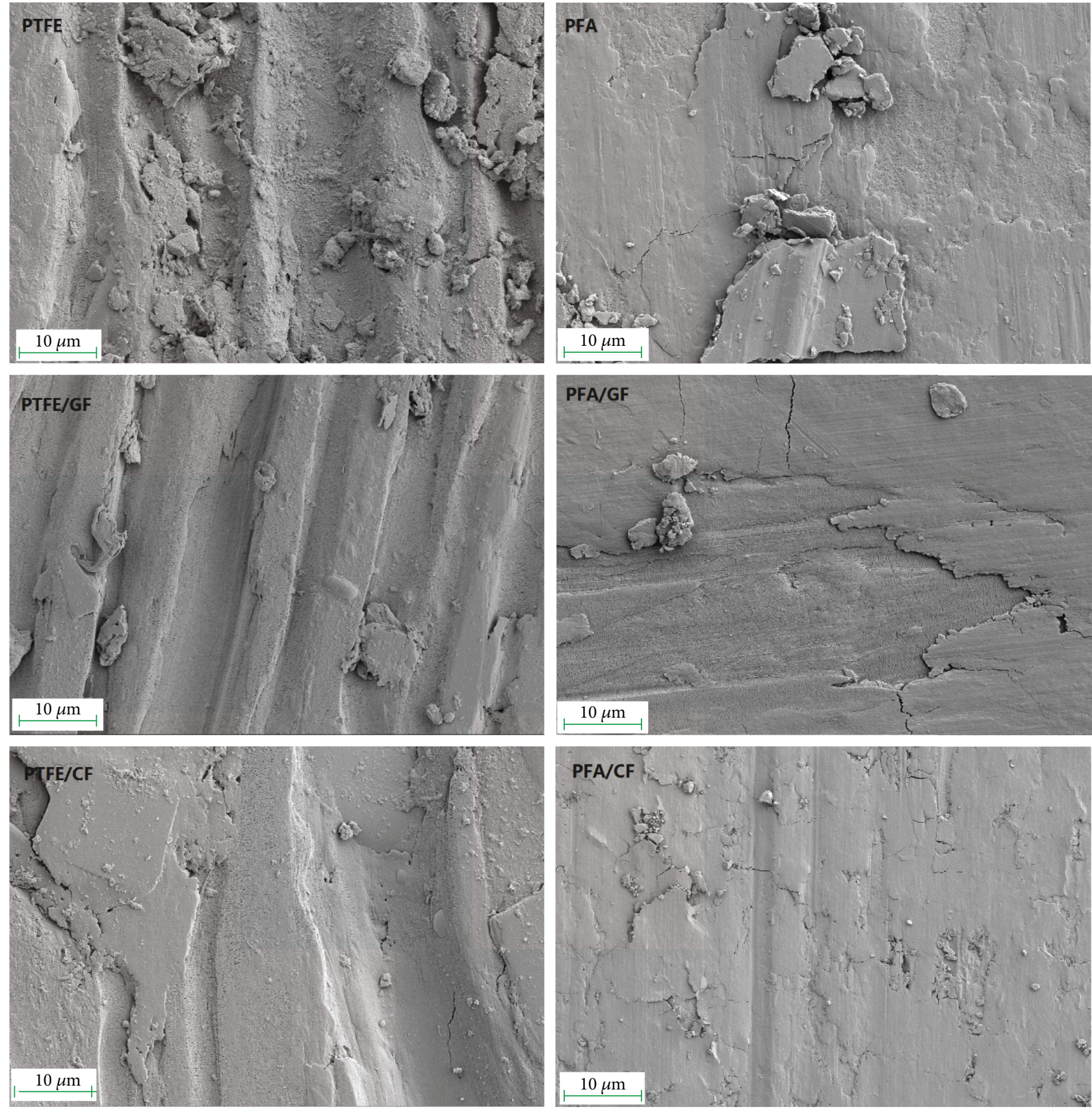

FIGURE 5: SEM worn surfaces of the PTFE and PFA composites.

robust transfer film is the key to reducing wear rate in a PFA composite.

\subsection{Wear and Seal Condition}

3.2.1. Wear Loss of the Seal. The level of wear loss on the step seals is shown in Figure 7. Generally, the results are very good in confirming the applicability of the composite materials for service under very demanding conditions. The most consistently high wear resistance is seen in PFA/CF seals, which demonstrates excellent performance with less than $0.1 \mathrm{wt} . \%$, while the weight loss of PTFE/CF seals is $0.5 \mathrm{wt} . \%$. The higher wear resistance of PFA/CF seals is possibly related to the material which may generate transfer films in the interface and thus prolong the lifetime of the seal.

3.2.2. Leakage of Seals. Figure 8 illustrates examples of how the accumulated leakage develops over time for PTFE/CF and PFA/CF seals. The leakage of PFA/CF seals is less than $200 \mathrm{~g}$ while that of PTFE/CF seals are greater than $1000 \mathrm{~g}$ after a $110 \mathrm{~km}$ working distance. Leakage is due to seal deformation and wear losses. The PFA/CF composite shows a better wear resistance than the PTFE/CF composite. This allows for less leakage when used as a lip seal for dynamic 


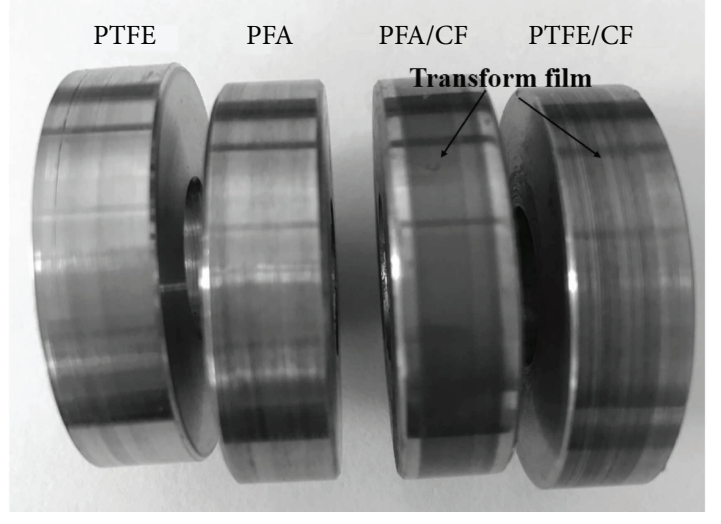

Figure 6: Transfer film of the PTFE and PFA materials on the couple rings.

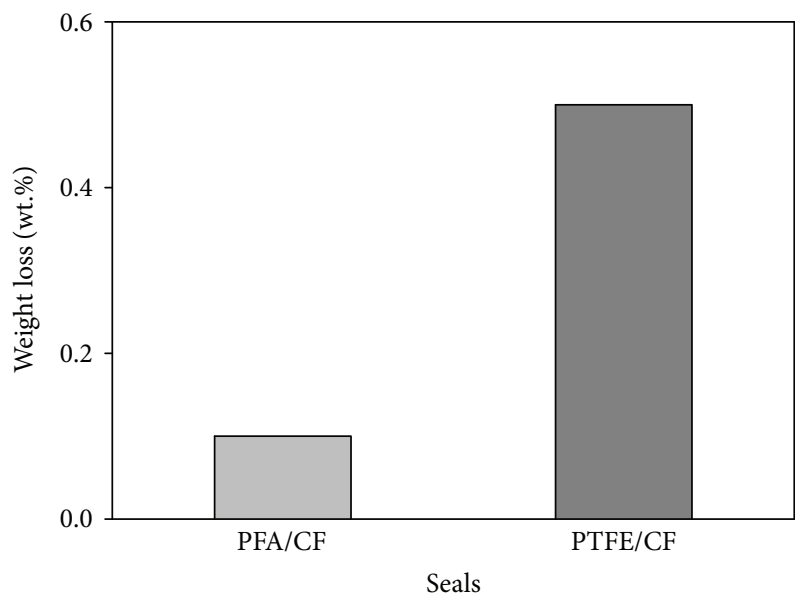

Figure 7: The weight loss of PTFE and PFA seal wear after test rigs.

shaft seals, thus prolonging the lifetime of the seal's function. This performance makes the PFA/CF seal a potential replacement for PTFE composite seals in many applications. PFA/CF sealing elements can be either machined or injection-molded, which makes it economically viable for both small quantity, short delivery requirements and high volume applications.

\section{Conclusions}

(1) The addition of glass fiber and carbon fiber as fillers increases the wear resistance and coefficient of friction of PTFE and PFA. Significant improvement in wear resistance is observed in PFA composites especially at high temperatures. PFA composites exhibited considerably smoother surfaces than PTFE composites.

(2) PTFE and PFA composites can facilitate the formation of transfer films on the steel ring. The transfer film of PFA/CF composites is much more unique,

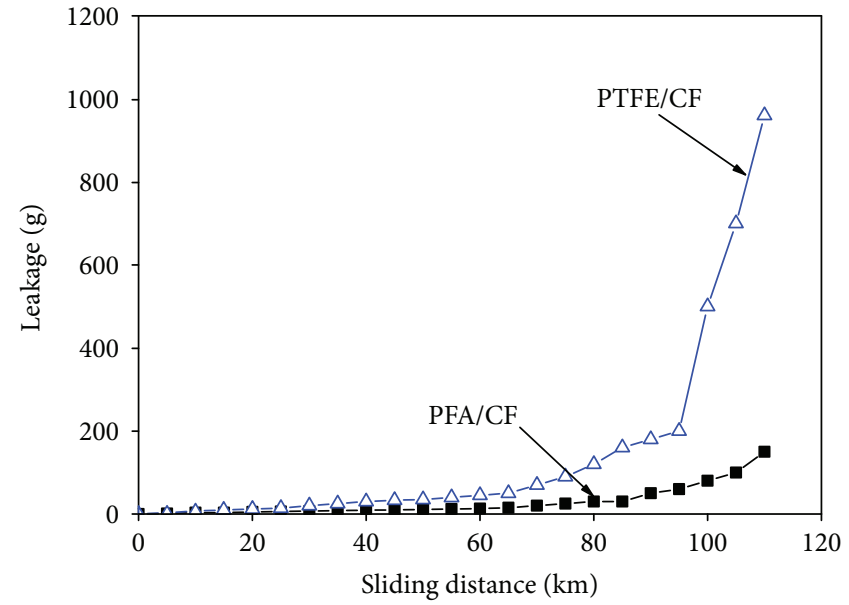

FIgure 8: Leakage of PTFE/CF and PFA/CF seals with sliding distance.

thick, smooth, and compact when compared to PTFE/CF composites.

(3) The weight loss and leakage of PFA/CF seals are significantly lower than those of PTFE/CF seals. PFA/CF seals are a potential replacement for PTFE composite seals for various applications.

\section{Conflicts of Interest}

The authors declare that they have no conflicts of interest.

\section{Acknowledgments}

This work was financially supported by Pearl River S\&T Nova Program of Guangzhou (the investigation of tribological properties of PTFE seals at high temperature and velocity) (201506010055) and Natural Science Foundation of Guangdong Province, China (2017A030310622).

\section{References}

[1] B. A. Krick, J. J. Ewin, G. S. Blackman, C. P. Junk, and W. Gregory Sawyer, "Environmental dependence of ultralow wear behavior of polytetrafluoroethylene (PTFE) and alumina composites suggests tribochemical mechanisms," Tribology International, vol. 51, pp. 42-46, 2012.

[2] J. Ye, H. S. Khare, and D. L. Burris, "Transfer film evolution and its role in promoting ultra-low wear of a PTFE nanocomposite," Wear, vol. 297, no. 1-2, pp. 1095-1102, 2013.

[3] J. R. Vail, D. L. Burris, and W. G. Sawyer, "Multifunctionality of single-walled carbon nanotube-polytetrafluoroethylene nanocomposites," Wear, vol. 267, no. 1-4, pp. 619-624, 2009.

[4] S. Ye, X. Zeng, F. Tan, and Q. Fan, "Research on the tribological performance of $\mathrm{Cr}_{2} \mathrm{O}_{3}$ filled with bronze-based PTFE composites," Journal of Applied Polymer Science, vol. 131, no. 22, 2014.

[5] R. C. Bowers and W. A. Zisman, "Frictional properties of tetrafluoroethylene-perfluoro (propyl vinyl ether) copolymers," Industrial \& Engineering Chemistry Research, vol. 13, no. 2, pp. 115-118, 1974. 
[6] M. A. Sidebottom, A. A. Pitenis, C. P. Junk et al., "Ultralow wear perfluoroalkoxy (PFA) and alumina composites," Wear, vol. 362-363, pp. 179-185, 2016.

[7] S. W. Zhang, "State-of-the-art of polymer tribology," Tribology International, vol. 31, no. 1-3, pp. 49-60, 1998.

[8] S. Bhargava and T. A. Blanchet, "Unusually effective nanofiller a contradiction of microfiller-specific mechanisms of PTFE composite wear resistance?," Journal of Tribology, vol. 138, no. 4, article 042001, 2016.

[9] M. Qiu, Y. Miao, Y. Li, and J. Lu, "Film-forming mechanisms for self-lubricating radial spherical plain bearings with hybrid PTFE/aramid fabric liners modified by ultrasonic," Tribology International, vol. 87, pp. 132-138, 2015.

[10] S. S. Kandanur, M. A. Schrameyer, K. F. Jung, M. E. Makowiec, S. Bhargava, and T. A. Blanchet, "Effect of activated carbon and various other nanoparticle fillers on PTFE wear," Tribology Transactions, vol. 57, no. 5, pp. 821-830, 2014.

[11] T. A. Blanchet, Y.-L. Peng, and S. V. Nablo, "Tribology of selectively irradiated PTFE surfaces," Tribology Letters, vol. 4, no. 1, pp. 87-94, 1998. 


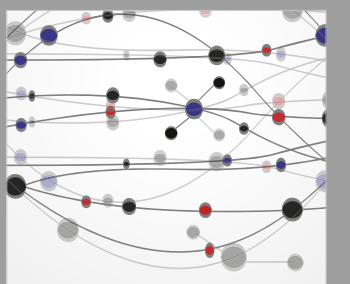

The Scientific World Journal
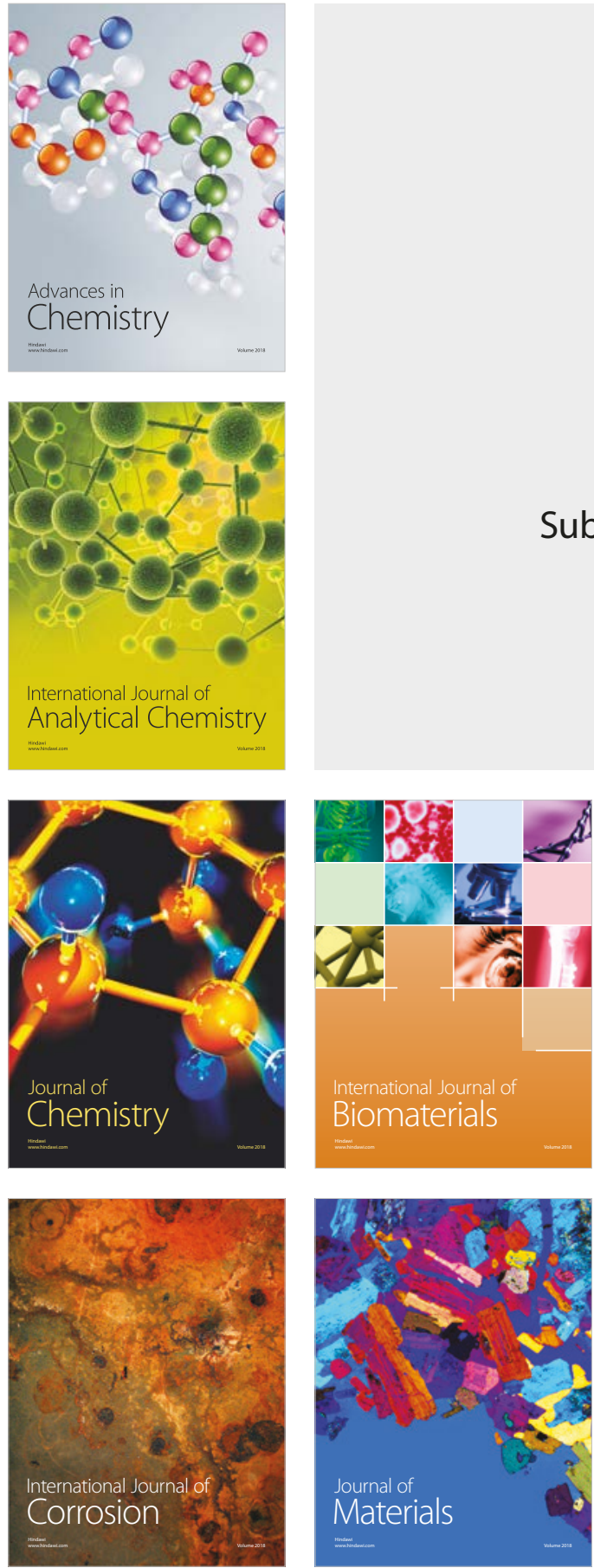

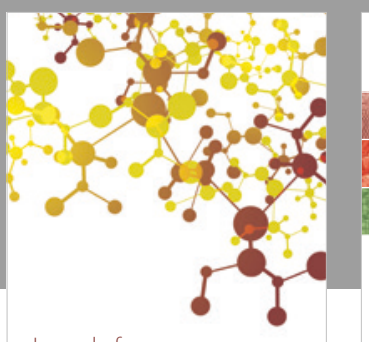

Journal of

Applied Chemistry
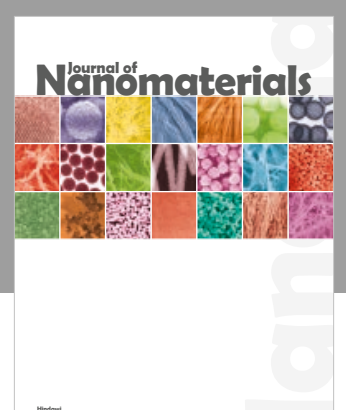

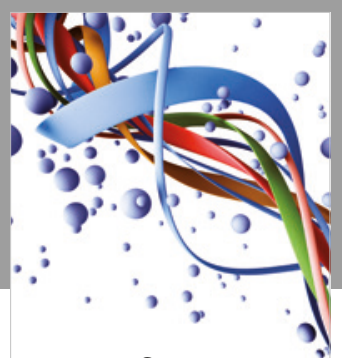

Scientifica

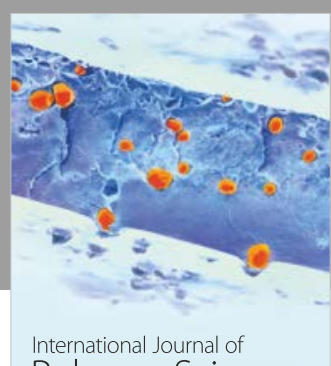

Polymer Science

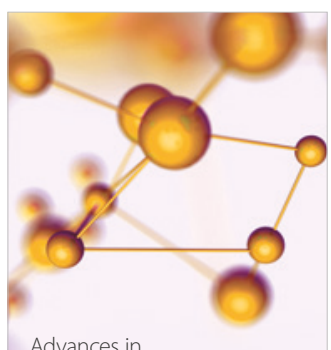

Physical Chemistry
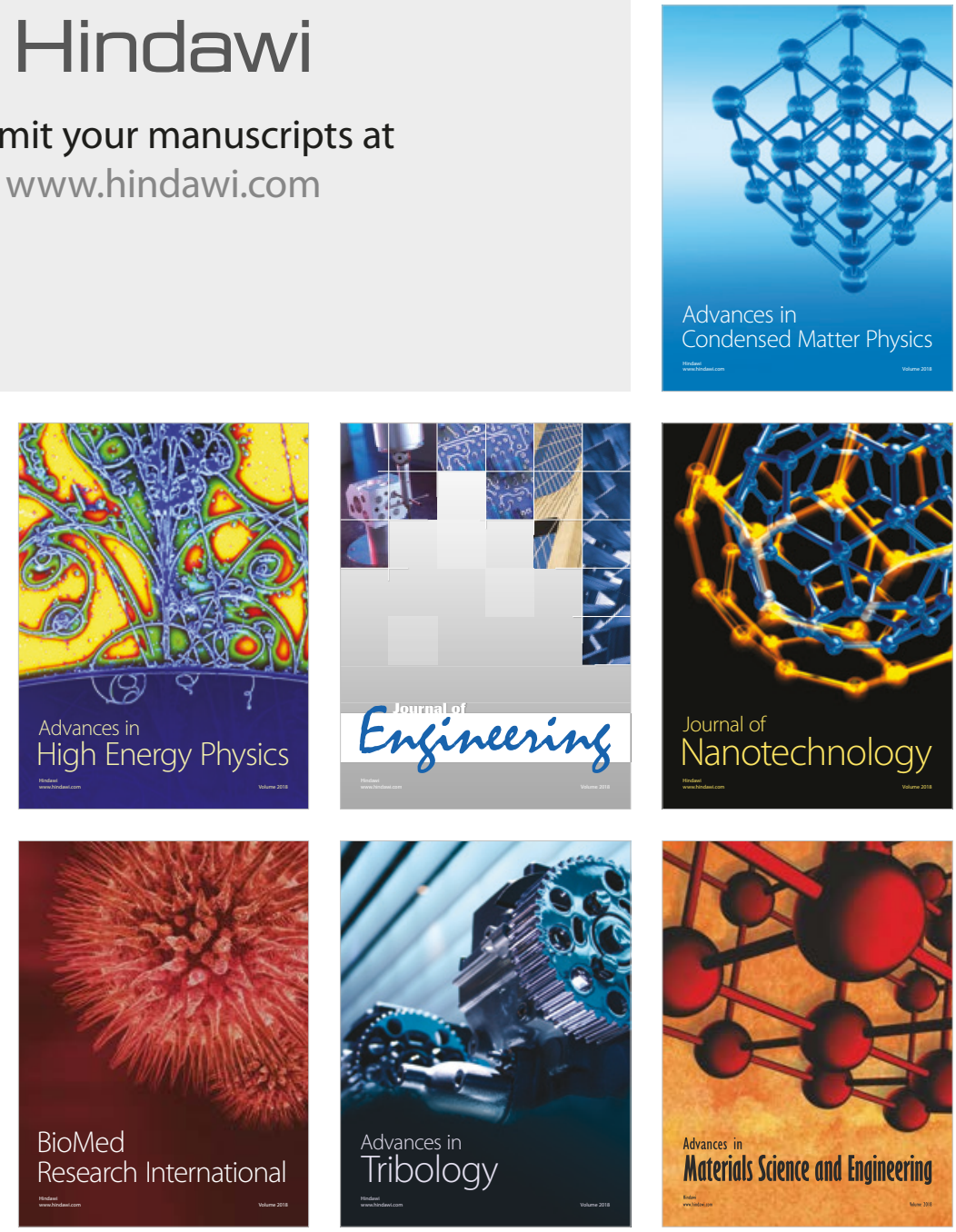